\title{
FACTORING A LATTICE ${ }^{1}$
}

\section{ALEXANDER DONIPHAN WALLACE}

For the purpose of this note a lattice is a Hasdorff space $L$ together with a pair $\wedge, \vee: L \times L \rightarrow L$ of continuous functions satisfying the usual conditions $[3$, p. 18]. The closed unit interval $I$ with the operations $\min , \max$ is a simple example. An iseomorphism is a simultaneous isomorphism and homeomorphism (A. H. Clifford). It is known and easy to prove [1] that a closed and bounded interval admits a unique (modulo an iseomorphism or a dual iseomorphism) structure. It is known [1] that a 2-cell admits more than one lattice structure. We shall show that if no maximal chain cuts $L$ into more than two pieces then the lattice structure is unique.

If $L_{1}$ and $L_{2}$ are lattices then $L_{1} \times L_{2}$ is the cartesian product of $L_{1}$ and $L_{2}$ with coordinate operations.

THEOREM. If the lattice $L$ is homeomorphic with a closed 2-cell and if no maximal chain cuts $L$ into more than two components then $L$ is iseomorphic with $I \times I$.

The proof is made by a series of lettered assertions.

(A) L has a zero and unit contained in its boundary [4].

Let $S$ and $T$ be the two arcs of the boundary of $L$ with endpoints 0 and 1.

(B) The arcs $S$ and $T$ are maximal chains.

Proof. Here and later it is suggested that a simple figure will enhance the geometric flavor of the argument. Let $x, y \in T$ with $0<x$, $y<1$, let $x$ precede $y$ on $T$ in the order from 0 to 1 and assume that $x \in L \backslash y \wedge L$, i.e., it is false that $x \leqq y$. Then $y \wedge L$ is compact and connected (continuous image of $L$ ), $0, y \in y \wedge L$ and $x, 1 \in L \backslash y \wedge L$. Thus $y \wedge L$ separates $x$ and 1 on the boundary of $L$ and thus (as is wellknown, [5]) $y \wedge L$ separates $L$. Hence $L \backslash y \wedge L=U \cup V$ where $U$ and $V$ are disjoint open sets with $1 \in V$. If $a \in U$ then $a \bigvee L$ is a connected set containing $a$ and 1 so that $(y \wedge L) \cap(a \vee L) \neq \square$. But if $b$ is in this set then $a \leqq b \leqq y$ so that $a \in y \wedge L$, a contradiction. Hence $T$ is a chain, i.e., $x, y \in T$ gives $x \leqq y$ or $y \leqq x$. Now let $C$ be a maximal chain containing $T$. Since $L$ is compact and connected it is easy to see that $C$ contains no proper connected subset containing 0 and 1 . Indeed, $C$ is an arc from 0 to $1[1]$. Hence $T=C$ and the proof is complete.

Received by the editors August 19, 1957.

1 This work was supported by the National Science Foundation. 
(C) The arcs $S$ and $T$ generate $L$ in the sense that $L=S \wedge T=S \vee T$.

Proof. Since $1 \in S \cap T$ we have $S \cup T \subset S \wedge T$ and $T \wedge(S \cup T)$ $\subset T \wedge S$. If $L \neq T \wedge S$ let $f$ be a function retracting $T \wedge S$ onto $T \cup S$, and define $g: T \times(T \cup S) \rightarrow T \cap S$ by $g(t, x)=t \wedge x$. Now $f g(0, x)=f(0)$ $=0$ and $f g(1, x)=f(x)=x$ since $x \in T \cup S$. The function $f g$ is then a homotopy between the identity and the constant map on $T \cup S$. This involves a contradiction.

(D) There are elements $b \in T \backslash\{0,1\}$ and $b^{\prime} \in S \backslash\{0,1\}$ such that $b \wedge b^{\prime}=0$ and $b \vee b^{\prime}=1$.

Proof. Let

$$
A=\{x \mid x \wedge L \subset T\} \text { and } B=\{x \mid x \vee L \subset T\}
$$

and note that $A$ and $B$ are closed and $x \in A$ gives $x \in x \wedge L \subset T$ so that $A \cup B \subset T$. If $a$ is the least upper bound of $A$ and if $b$ is the greatest lower bound of $B$ we show that $b \leqq a$. This being false let $p \in T$ such that $a<p<b$ (see (B)). Then $p \wedge L$ is not a part of $T$ so let $a_{0} \in p \wedge L \backslash T$. Then $a_{0}=t \bigvee a_{1}$ with $t \in T$ and $a_{1} \in S$. If $a_{1}=0$ then $a_{0} \in T$. Thus $0<a_{1}<p$ since $a_{1} \leqq p$ and $a_{1}=p$ gives $a_{0} \in T \vee T \subset T$, by (B). In a dual fashion we show that $p<b_{1}<1$ for some $b_{1} \in S$. Let $C$ be a maximal chain containing $0, a_{1}, p, b_{1}$ and 1 so that $C$ is an arc in $L$ from 0 to 1 [1]. A figure will indicate that $L \backslash C$ has more than two components, a fact that can be verified by applying known results on $\theta$-curves [5, Chap. VI]. It can also be proved in consequence of the Jordan Theorem and the fact that a spanning arc of a 2-cell cuts the 2-cell, loc. cit. Since this contradicts our hypotheses we infer that $b \leqq a$.

If $b=1$ then $a=1$ so that $L=a \wedge L \subset T$, an impossibility. Were $b=0$ then $L=b \bigvee L \subset T$, so that $0<b<1$. Now $x \leqq y \in A$ gives $x \in A$ so that $b \in A$ and hence $(b \wedge L) \cup(b \bigvee L) \subset T$. But $0,1 \in(b \wedge L) \cup(b \bigvee L)$ and this set being connected we have $(b \wedge L) \cup(b \backslash L)=T$. Dually, we locate $b^{\prime} \in S$ with $0<b^{\prime}<1$ and $\left(b^{\prime} \wedge L\right) \cup\left(b^{\prime} \vee L\right)=T$. From $S \cap T$ $=\{0,1\}$ an easy computation leads to $b \wedge b^{\prime}=0$ and $b \vee b^{\prime}=1$ and (D) is proved.

(E) The function $f: L \rightarrow(b \wedge L) \times\left(b^{\prime} \wedge L\right)$ defined by $f(x)=(b \wedge x$, $\left.b^{\prime} \wedge x\right)$ is an iseomorphism.

Proof. By a result of $\mathrm{L}$. W. Anderson, we know that $L$ is distributive [2]. The arguments in $\S 9$, Chap. II of [3] and the fact that $L$ is compact complete the proof.

The proof of the theorem is finished if we note that $b \wedge L$ and $b^{\prime} \wedge L$ are chains and hence arcs and so have the structure of $I$.

The content of the theorem is really the assertion that $L$ has a nontrivial center [3, p. 27] and (as pointed out to me by R. D. Ander- 
son) it may be observed that the center of $L$ has cardinal four. It has been conjectured that the center of any compact connected $n$-dimensional lattice has cardinal at most $2^{n}$.

L. W. Anderson ([1], to appear in Proc. Amer. Math. Soc.) has shown that a compact connected 1-dimensional lattice is a chain. It is an easy conjecture that a compact connected 2-dimensional metrizable lattice without cutpoints is a 2-cell. If it can be imbedded in the plane then it is a 2-cell. A proof of this fact (which is quite easy) will appear in the Pacific Journal of Mathematics. Thus, in the theorem of this note, the hypothesis that $L$ be a 2 -cell could be replaced by the assumptions that $L$ is compact, connected, imbeddable in the plane and without cutpoints.

It is noted that (A), (B), and (C) are valid without the maximal chain condition on $L$. In fact, these results can be reformulated so as to be valid for semigroups.

A. L. Shields obtained a result either identical with, or closely related to, a suitable reformulation of $(\mathrm{C})$.

\section{BiBLIOGRAPHY}

1. L. W. Anderson, Topological lattices, Tulane University Dissertation, 1956.

2. - The distributivity and simple connectivity of plane topological lattices, to appear in Trans. Amer. Math. Soc.

3. G. Birkhoff, Lattice theory, New York, 1948.

4. A. D. Wallace, The peripheral character of central elements of a lattice, Proc. Amer. Math. Soc. vol. 8 (1957) pp. 596-597.

5. G. T. Whyburn, Analytic topology, New York, 1942.

The Tulane University of Louisiana 